\title{
Medical Problems at Birth and Death ${ }^{* *}$
}

\author{
Kentaro TAKagi, MD*
}

\section{Brain death}

The idea that stoppage of the heart beat, dilatation of the pupils and cessation of respiratory movements are the decisive triad for a person's death has been fixed in the field of medical care over the past several centuries. On the basis of this principle, medical providers have declared the patient's death without any doubt.

However, great advances in life maintenance apparatuses in recent years has enabled us to keep the patient's body alive, except for the brain, for more than several days after the irreversible death of all brain stem functions including cessation of respiratory movements and disappearance of light reflex. This has inevitably resulted in the introduction of a new concept of death, i.e., "brain death."

It cannot be denied that progress in organ transplantation underlies the fact that advanced countries have been compelled to approve brain death medically and legally for the past 10 years or so. To give a part of the body that will never revive as a human to another person in need is a very effective, rational act, which would be equivalent to offering our own bodies for the sake of medical education. However, to extirpate the organs from a dead person who appears to be still alive is very loathsome from the emotional aspect not only for his or her relatives, but also for doctors. There are also some unsolved problems, such as the objection that brain death is not the death of an individual but is partial death involving the death of the brain and ambiguity of the time to declare death. In consideration of the present state that organ transplantation is acutally performed in the condition of brain death, it is essential to legally establish brain death as death in Japan. For this purpose, it is necessary to establish the criteria for brain death among doctors or in the field of medical sciences as soon as possible and to strive for national consensus in this regard.

\section{Organ transplantation}

Great progress in transplantation and the discovery of cyclosporin A have reduced immunorejection, which is an obstacle to success in transplantation. The resulting improvement in the fixation rate of transplanted organs and decrease in postoperative infections disease have resulted in a rapid increase in the survival rate. Now, cardiac, cardiopulmonary, pulmonary, hepatic and pancreatic transplantations are performed in advanced countries to the great advantage of many patients. In contrast, in Japan renal transplantation alone is performed. In most cases, the kidney is transplanted from the living body, and half of the transplantable kidneys from dead bodies are provided by the USA. Furthermore, the present situation is that patients requiring cardial transplantation go to a hospital in the USA for such a transplantation.

At present, real organs are far superior to artificial ones. Therefore, it is strongly desired to pay high regard to the scientific and rational spirit that transplantable organs should be used effectively by giving them to patients seriously in need who cannot live without them in order to prolong their lives, rather than disposing of them in vain.

\section{Euthanasia}

Even in patients with intense pain, such as those in the terminal stage of cancer, which makes them wish for death, prolongation of life with every possible method for relieving pain should be placed before all other things. I object

\footnotetext{
*Emeritus Professor of Nagoya University and Nagoya City University.

**Presented at the 81 st Annual Meeting of the Japanese Society of Internal Medicine, April 2, 1984, in Fukuoka.
} 
to the thoughtless application of euthanasia. I cannot approve such death either, in principle, even with a living will, in a so-called human vegetable in whom all animal functions have disappeared, as long as the brain stem is functioning. However, when the disease persists for an extremely long period, the burden becomes too heavy for the family of the patient from the physical, mental and economic aspects, and no possible medical procedure can make the patient recover, active treatment should be discontinued according to the living will on the basis of the consent of the family, in order to allow the patient to die peacefully.

\section{Artificial termination of pregnancy}

The ovum more than 16 days after fertilization cannot grow if it is cut into two pieces. Therefore, the fertilized ovum is already an organism at this period. But not a human. In the fetus, blood circulation develops at one month of gestation, digestive and excretory functions at two months. Regular respiratory movements begin from the 13th week (about three months) of gestation. This means that the brain stem has already begun to function at this period.

If we take the position of attaching considerable importance to the view that the ovum even just after fertilization is a living thing that will be a human in the future, it is not allowable to take its life away. However, I think that artificial abortion can be approved within 13 weeks of gestation, i.e., before the appearance of the brain stem function, which is the minimum condition for a human life, provided that the mother has a serious reason for requiring it.

Artificial abortion between three and six months based on simple economic considerations should not be approved, unless the mother's health is endangered.

At seven months of gestation, the fetus, which already has sufficient autonomy, is a complete human.

\section{External fertilization}

It is desired that discussions on moral regulations for external fertilization be first carried out sufficiently in medical circles to prepare a unified opinion. In Japan, things are currently going in such a direction. General opinion approves external fertilization between a married couple. However, increasing internationalization and the fact that various types of external fertilization are being performed in European and American countries are taken into consideration, it is suggested that people who desire to have their own children go to other countries to realize their desire if it is impossible in Japan. It may be necessary to consider in advance possible moves to make in such a case. 